\title{
Optimizing F-actin Labeling At the Leading Edge Of Cells Using Multiple Actin Probes, Fixation Methods and Imaging Techniques
}

\author{
Vera DesMarais ${ }^{1,2,3}$, Robert J. Eddy ${ }^{1}$, Ved P. Sharma ${ }^{1}$, Orrin Stone ${ }^{4}$ and John S. Condeelis ${ }^{1,2,3}$ \\ 1. Department of Anatomy and Structural Biology/Albert Einstein College of Medicine, Bronx, NY, USA. \\ 2. Analytical Imaging Facility, Albert Einstein College of Medicine, Bronx, NY, USA. \\ 3. Gruss-Lipper Biophotonics Center, Albert Einstein College of Medicine, Bronx, NY, USA. \\ 4. Department of Pharmacology, UNC Chapel Hill School of Medicine, Chapel Hill, NC, USA.
}

Actin is a highly abundant, conserved protein in eukaryotic cells that exists in two principal forms, globular, monomeric (G) actin, and polymerized filamentous (F) actin. Along with intermediate filaments and microtubules, actin filaments (F-actin) comprise the cytoskeleton of eukaryotic cells. Actin polymerization is used by many cells as the driving force for directed cell migration [1,2]. In the cell periphery, F-actin is present in the sub-membranous lamellipodium, where it consists of a dynamic population of relatively short filaments, and in the adjacent lamella, where it consists of longer, unbranched filaments stabilized by tropomyosin $[3,4]$. F-actin has a highly dynamic nature, therefore acquiring accurate and detailed images of actin in both live and fixed cells can be challenging. The quality of images depends directly on the quality of the sample preparation, especially for high-resolution imaging techniques such as confocal and super-resolution microscopy.

Our study critically evaluates and compares commercially available, commonly used fixation protocols and probes for labeling F-actin in a highly-motile breast adenocarcinoma cell line (MTLn3) to optimize the visualization of F-actin in dynamic lamellipodia. This will guide the end user in making rational choices with respect to which actin-probe to use. We evaluated four Phalloidin-dye conjugates [5], two anti-actin antibodies, and three live-cell actin probes [6,7], using five fixation conditions with three different imaging modalities in order to develop optimized protocols.

Of the four fluorescent Phalloidin-dye conjugates tested, Alexa Fluor-488 Phalloidin ranked highest in the persistence of the fluorescent signal over time and in revealing fine structures of the actin cytoskeleton visualized by Structural Illumination Microscopy (SIM), when compared to either wide-field epifluorescence microscopy or point-scanning confocal microscopy. The optimization of a fixation protocol for studying dynamic actin filaments in the lamellipodia of highly motile cells requires a trade-off between speed, morphology and antigen preservation. Therefore, in order to maximize the quality of staining, we examined several fixation methods, including aldehydes and organic solvents, with sequential and simultaneous fixation protocols. We found the Simultaneous Form/Glut/Saponin protocol superior in the staining of F-actin in lamellipodia.

Staining by actin monoclonal antibodies (AC-15 and AC-40) did not show the same level of detail of actin structures compared to Phalloidin-staining under a variety of fixation-permeabilization conditions. Evaluation of commonly used live-cell actin probes showed evidence for actin filament bias, with TagRFP-Lifeact excluded from lamellipodia, but not mEGFP-Lifeact or F-tractin-EGFP. This suggests that the cellular distribution of individual Lifeact fluorescent protein reporters does not always accurately represent the actin cytoskeleton in a given cell type.

After evaluating three different F-actin labeling techniques, along with a variety of different fixation 
techniques, Phalloidin- 488 was judged to be the most robust method of labeling the detailed structures of the actin cytoskeleton. The actin cytoskeleton appeared to be preserved best by rapid, simultaneous Form/Glut/Saponin fixation with SIM imaging, see Figure 1 [8].

\section{References:}

[1] CM Franz, GE Jones and AJ Ridley, Dev Cell 2 (2002), p. 153.

[2] J Condeelis, Annu Rev Cell Biol 9 (1993), p. 411.

[3] TM Svitkina and GG Borisy, J Cell Biol 145 (1999), p. 1009.

[4] V DesMarais et al., Journal of Cell Science 115 (2002), p. 4649.

[5] J Vandekerckhove et al., EMBO 4 (1985), p. 2815.

[6] J Riedl et al., Nat Methods 5 (2008), p. 605.

[7] BJ Belin, LM Goins and RD Mullins, Bioarchitecture 4 (2014), p. 189.

[8] All imaging was conducted in the Analytical Imaging Facility (AIF) (funded by NCI Cancer Grant P30CA013330). SIM imaging was executed on the Nikon-STORM/SIM/TIRF microscope (funded by NIH 1S10OD18218-1). Some confocal imaging was executed on the Leica SP8 confocal microscope (funded by NIH 1S10OD023591-01). The research was funded by grant CA150344 (RE, VS, JC).

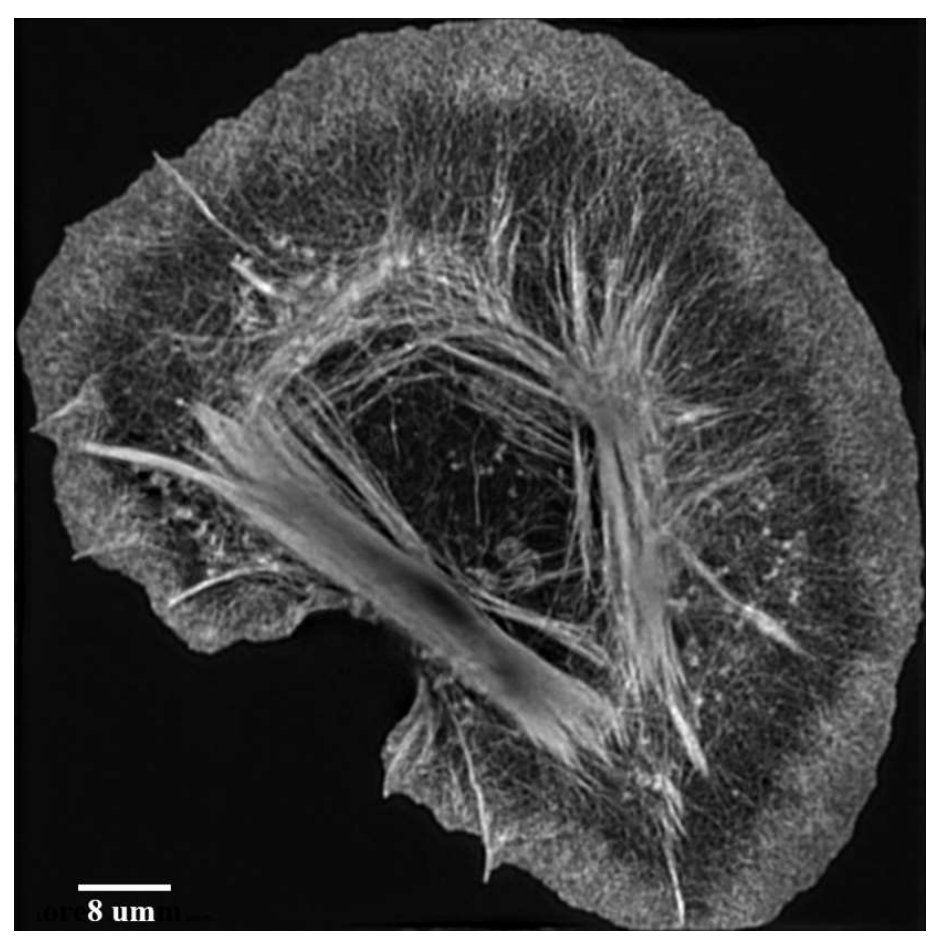

Figure 1. The most highly detailed F-actin filaments were revealed by super-resolution Structural Illumination Microscopy (SIM) of Phalloidin-488 labeled cells with simultaneous Form/Glut/Saponin fixation. MTLn3 cells were treated with EGF for 3 min, fixed with simultaneous Form/Glut/Saponin fixation and stained with Phalloidin-488. Image was acquired by SIM and displayed as maximal pixelprojections of a z-stack. 